\title{
Desordens hematológicas autoimunes e infecção pelo SARS-CoV-2: revisão de literatura
}

\author{
Autoimmune hematologic disorders and SARS-CoV-2 infection: literature review
}

Trastornos hematológicos autoinmunes y infección por SARS-CoV-2: revisión de la literatura

Julius Caesar Mendes Soares Monteiro ${ }^{1,2 *}$, Hiroyuki Otsuki Guimarães ${ }^{3}$, Bruno Pinto de Oliveira ${ }^{3}$, Herbert Paulino Cordeiro², Fernanda de Nazaré Cardoso dos Santos Cordeiro², Fernando Costa Araújo ${ }^{1,2}$, Leonardo Teixeira de Mendonça².

\section{RESUMO}

Objetivo: Descrever as desordens hematológicas imunomediadas secundárias à infecção pelo SARS-CoV2, explorando tanto aspectos fisiopatológicos quanto propostas de tratamento. Revisão bibliográfica: $A$ infecção pelo SARS-CoV-2 está relacionada a diversas desordens do sistema hematológico, sejam precipitadas pela ativação da autoimunidade (ativação do sistema complemento, ativação de respostas celulares imunológicas) ou por efeito direto do vírus no endotélio; podendo estar relacionadas a alterações em marcadores inflamatórios ou de coagulação, que podem ser considerados na busca diagnóstica de tais manifestações, além de estados pro trombóticos e acometimentos orgânicos locais ou sistêmicos. Dentre as principais manifestações descritas têm-se: Síndrome de Evans, Anemia Hemolítica Autoimune, Síndrome do Anticorpo Antifosfolípide, Púrpuras imunológicas e o aumento na relação neutrófilo/linfócito. Considerações finais: Considerando a pertinência da COVID-19 como desafio de saúde internacional, bem como a falta de terapêutica específica, é de suma importância o conhecimento sobre as diversas comorbidades que podem se associar, em especial aos fenômenos tromboembólicos, devido sua expressiva morbimortalidade e possibilidade de prevenção.

Palavras-chave: COVID-19, Desordens hematológicas autoimunes, Doenças autoimunes.

\begin{abstract}
Objective: To describe immune-mediated hematological disorders secondary to SARS-CoV-2 infection, exploring both pathophysiological aspects and treatment proposals. Bibliographic review: The infection by SARS-CoV-2 is related to several disorders of the hematological system, whether triggered by the activation of autoimmunity (activation of the complement system, activation of cellular immune responses) or by the direct effect of the virus on the endothelium; they may be related to changes in inflammatory markers or coagulation, which can be considered in the diagnostic search for such manifestations, in addition to prothrombotic states and local or systemic organic involvement. Among the main manifestations described are: Evans Syndrome, Autoimmune Hemolytic Anemia, Antiphospholipid Antibody Syndrome, Immunological purpura and the increase in the neutrophil/lymphocyte ratio. Final considerations: Considering the relevance of COVID-19 as an international health challenge, as well as the lack of specific therapy, it is extremely important to know about the various comorbidities that can be associated, especially to thromboembolic phenomena, due to its expressive morbidity and mortality and possibility of prevention.
\end{abstract}

Keywords: COVID-19, Autoimmune hematological disorders, Autoimmune diseases.

\footnotetext{
${ }^{1}$ Hospital Universitário João de Barros Barreto (HUJBB), Belém - PA.

*E-mail:julius@ufpa.br

${ }^{2}$ Centro Universitário Metropolitano da Amazônia (UNIFAMAZ), Belém - PA.

3 Universidade Federal do Pará (UFPA), Belém - PA.
} 


\section{RESUMEN}

Objetivo: Describir los trastornos hematológicos inmunomediados secundarios a la infección por SARS-CoV2, explorando tanto los aspectos fisiopatológicos como las propuestas de tratamiento. Revisión bibliográfica: La infección por SARS-CoV-2 está relacionada con varios trastornos del sistema hematológico, ya sea provocados por la activación de la autoinmunidad (activación del sistema del complemento, activación de las respuestas inmunitarias celulares) o por el efecto directo del virus sobre el endotelio; pueden estar relacionados con cambios en los marcadores inflamatorios o en la coagulación, lo que puede ser considerado en la búsqueda diagnóstica de tales manifestaciones, además de estados protrombóticos y afectación orgánica local o sistémica. Entre las principales manifestaciones descritas se encuentran: Síndrome de Evans, Anemia hemolítica autoinmune, Síndrome de anticuerpos antifosfolípidos, Púrpura inmunológica y el aumento de la relación neutrófilos / linfocitos. Consideraciones finales: Considerando la relevancia del COVID-19 como un desafío de salud internacional, así como la falta de una terapia específica, es de suma importancia conocer las diversas comorbilidades que se pueden asociar, especialmente a los fenómenos tromboembólicos, por su expresiva morbilidad. y mortalidad y posibilidad de prevención.

Palabras clave: COVID-19, Enfermedades hematológicas autoinmunes, Enfermedades autoinmunes.

\section{INTRODUÇÃO}

Iniciou-se a partir da cidade de Wuhan (China), desde o final de 2019, uma epidemia de pneumonia atípica que, posteriormente, foi identificada como de origem viral presumidamente zoonótica. Foi então designada pela Organização Mundial da Saúde (OMS) de doença causada pelo novo coronavírus-19 (COVID-19) à semelhança de outros causadores de síndrome respiratória aguda grave (SRAG) de caráter epidêmico. (TERPOS E, et al., 2020; SMADJA DM, et al., 2020). O SARS-CoV-2 é uma espécie viral da família dos coronavírus (CoV's), apresentando características de vírus envelopados de fita simples positiva de RNA; podendo apresentar capsídeos pleomórficos de projeções radiais superficiais como uma coroa, originando o nome "coronavírus" (XAVIER AR, et al., 2020).

Atualmente, com status de pandemia global, a COVID-19 vem apresentando diferentes padrões clínicoepidemiológicos a depender do período e região analisada; atualmente em estabilização global no número de casos confirmados, porém com persistência no aumento de novos casos e óbitos no continente Europeu (OMS, 2021). Segundo a OMS, entre os dias 11/10/2021-17/10/2021, os casos confirmados de COVID-19 mantiveram-se com números semelhantes quando comparados com a semana anterior, com cerca de 2,75 milhões de novos casos reportados na semana; havendo decrescimento de tal registro globalmente, exceto pela região Européia com registros de crescimento de $7 \%$ em novos casos. Além disso, a persistência dos maiores valores de novos casos/óbitos mundiais, em valores brutos, está relacionada às regiões Americana e Européia. Especificamente, quanto ao Brasil, fora reportado desde o início da pandemia 21.644.464 casos confirmados e 603.282 óbitos (WHO, 2021).

Até o momento, identificaram-se diferentes evoluções clínicas no curso da infecção pelo SARS-Cov-2: portadores assintomáticos; Doença Respiratória Aguda (DRA); e ainda uma miríade de manifestações hematológicas, reumatológicas, cardiológicas e outras. A apresentação mais exuberante é na forma de pneumonia/sepse viral em diferentes graus de gravidade. Inicialmente, em pacientes sintomáticos, pode haver progressão de sintomas inespecíficos (febre, tosse, mialgia, fadiga, dor de garganta, diarreia, disgeusia, hiposmia/anosmia) até evolução para repercussões inflamatórias em trato respiratório inferior, culminando em casos mais graves da doença com dispneia severa, edema pulmonar e pneumonia viral grave. Os pacientes que apresentam esse comprometimento pulmonar mais severo podem necessitar de internação em leito clínico ou até em Unidades de Terapia Intensiva (UTI), com tratamento ventilatório de suporte por cateter nasal, máscaras com reservatório ou ventilação mecânica invasiva (SMADJA DM, et al., 2020; XAVIER AR, et al., 2020).

Alguns pacientes podem apresentar outros sintomas durante a fase inicial de COVID-19, como: secreção respiratória, rinorreia, dor de cabeça, hemoptise e diarreia. Houve relatos de casos que justificam atenção a 
indivíduos que apresentam comorbidades ou fatores de risco para evolução mais grave ou complicações sistêmicas, como a Síndrome do Desconforto Respiratório Agudo (SDRA) e Disfunção de Múltiplos Órgãos (DMOS). Algumas dessas comorbidades e fatores de risco a serem consideradas seriam: o Diabetes Mellitus (DM), doenças cardiovasculares e/ou renais, pacientes oncológicos, pacientes em uso de imunossupressores, idade a partir da $6^{\underline{a}}$ década de vida; podendo haver como sinais de alarme dor no peito, dispneia, linfopenia e aumento de indicadores inflamatórios (TERPOS E, et al., 2020; XAVIER AR, et al., 2020).

Os mecanismos exatos da infecção e patogenicidade do SARS-CoV-2, apesar de ainda não totalmente elucidados, permitem compreensão inicial de como poderiam surgir algumas das possíveis manifestações da doença. A hipótese mais aceita momentaneamente seria a interação da proteína spike do SARS-CoV-2 com receptores de enzima conversora de angiotensina 2 para a entrada na célula, a qual é expressa em diversos tecidos do corpo como: trato respiratório superior e inferior, trato gastrointestinal, cardiomiócitos, células do endotélio vascular e da microarquitetura renal; tal interação pode resultar em down regulation desses receptores, ou efeito citopático por indução direta do vírus culminando com morte celular e inflamação (AHMED S, et al., 2020; XU H, et al., 2020).

Além do mecanismo de infecção, a própria fisiopatologia da infecção precisa de maiores esclarecimentos. Pesquisas iniciais têm indicado diversas vias pelas quais o vírus pode causar dano ao corpo, alguns dos principais sintomas da infecção se relacionam a tempestade de citocinas (principalmente: IL-6, IL-2, IL-7, fator estimulador da colônia de granulócitos - G-CSF, interferon gama e o fator de necrose tumoral alfa - TNFa) que é lançada no corpo em função da replicação viral exacerbada que retroalimenta a cadeia, culminando em lise das células infectadas (LIN L, et al., 2020; MEHTA P, et al., 2020).

Tal lise celular inicial corrobora com as cascatas inflamatórias endógenas e aumenta a expressão de receptores inflamatórios, ativação de células imunológicas e quimiotaxia/ativação de linfócitos, bem como a apoptose celular desregulada. Soma-se a esse mecanismo, a lesão vascular direta, que pode ser observada tanto in vivo quanto in vitro, no endotélio infectado pelo SARS-CoV-2 (VARGA Z, et al., 2020). Esses dois mecanismos primários de lesão do SARS-CoV-2 permitem a compreensão da magnitude dos efeitos que o vírus pode desencadear em diversos sistemas corporais (CHANNAPPANAVAR R e PERLMAN S, 2017).

Fica claro, portanto, que apesar de reconhecidamente a lesão pulmonar ser causa de grande morbimortalidade nessa doença, existem diversas outras formas de acometimento orgânico que agem em sinergia e que podem servir de preditor de gravidade do quadro, bem como auxiliar no entendimento completo da situação do doente e garantir melhores chances de um tratamento guiado por fenótipos da doença. Neste sentido, o objetivo deste trabalho foi a descrição das principais desordens hematológicas autoimunes reconhecidas em pacientes com COVID-19, bem como o entendimento de seus potenciais mecanismos patogênicos e estratégias terapêuticas no manejo desta condição de alta prevalência na modernidade.

\section{REVISÃO BIBLIOGRÁFICA}

Os distúrbios do sistema hematopoiético se estabeleceram como parte do conjunto de sinais e sintomas da COVID-19 desde os primeiros casos relatados. Observou-se que muitos pacientes se apresentavam já na admissão com alterações hematológicas evidentes, ou desenvolviam ao longo da internação algum tipo de evento relacionado aos níveis eritrocitários, leuco/linfocitários e plaquetários, ou ainda eventos agudos relacionados a estados pró-trombóticos; bem como alterações em marcadores sanguíneos relacionados a inflamação ou à coagulação (SCHULMAN S, 2020).

As principais alterações relacionadas ao hemograma dizem respeito aos leucócitos e às plaquetas, com achados médios de linfopenia em $80 \%$ dos pacientes na fase aguda, variando de serviço a serviço; havendo leucopenia em até $30 \%$ e trombocitopenia em até $36 \%$ dos pacientes (WANG D, et al., 2020). Na leucometria, em especial de pacientes mais graves ou admitidos em UTI, verifica-se importante linfopenia; majoritariamente, às custas de linfócitos $\mathrm{TCD}^{8+}$ (LIPPI G e PLEBANI M, 2020).

Uma hipótese que tenta justificar tal achado se relaciona com a característica altamente imunogênica do SARS-CoV-2, ao invadir tecidos e causar lesões por lise ou endoteliais diretas, havendo grande recrutamento 
de linfócitos $T C D^{4+}$ nos tecidos que por sua vez levam a uma ativação inicialmente aumentada dos efetores $\mathrm{LTCD}^{8+}$. Esse fenômeno pode corroborar para a ocorrência da tempestade de citocinas, que rapidamente progride para exaustão linfocitária e à acidose láctica. A linfopenia, parece estar relacionada a maior taxa de internação em unidade de UTI, maior desenvolvimento de síndrome respiratória aguda grave e maior mortalidade (FAN BE, et al., 2020; LIPPI G e PLEBANI M, 2020).

Outra via para explicar a emergência da linfopenia reside na ativação macrofágica e não nos mecanismos intrínsecos do vírus. A contínua ativação de macrófagos, monócitos e a lesão endotelial são elementos que colaboram para o estado inflamatório sistêmico do paciente. O SARS-CoV-2 ativa células endoteliais quando interage com seus receptores da enzima conversora de angiotensina 2 (ECA-2), levando a liberação de citocinas de resposta rápida ativadoras de macrófagos (CCL-2 e CCL-7), além de moléculas ativadoras de liberação tardia como o interferon-1, responsáveis pelo recrutamento contínuo de monócitos, que por sua vez se diferenciam em macrófagos inflamatórios (RAHI MS, et al., 2020).

Tanto os linfócitos $T$ ativados quanto as células NK também recrutam macrófagos, através da liberação dos fatores estimuladores da colônia de granulócitos, TNFa e Interferon gama, aumentando ainda mais o recrutamento e a resposta inflamatória sistêmica induzida por macrófagos. Os Linfócitos que expressam a ECA-2 em suas membranas podem induzir a entrada do SARS-CoV-2 nos macrófagos derivados de monócitos, levando a uma liberação massiva de citocinas por parte dessas células, em especial IL-6, IL-8, IL10 e TNFa, gerando a tempestade de citocinas que caracteriza o estado inflamatório sistêmico da COVID-19 e perpetuando as vias de ativação leucocitária; que atua em sinergia aos demais fatores elencados acima para o estado pró-trombótico, e em última instância pode levar à síndrome hemofagocítica (IBA T, et al., 2020; XU H, et al., 2020).

Outro importante elemento leucocitário que sofre interferência são os neutrófilos, apresentando seu quantitativo aumentado na circulação periférica, podendo também estar diminuídos em alguns casos (LIPPI G e PLEBANI M, 2020). Surge então um importante índice que tem sido relacionado com pior desfecho para esses indivíduos: a Relação Neutrófilo/Linfócito (RNL). Tal preditor tem se mostrado importante marcador prognóstico para mortalidade, uma vez que em pacientes com um RNL aumentado na admissão, ou naqueles já internados e também na UTI, a chance de evolução desfavorável para óbito é maior em comparação ao grupo que apresenta leucograma normal ou mesmo alterado, porém sem aumento da relação RNL (TERPOS E, et al., 2020; RAHI MS, et al., 2020).

Ainda em relação aos elementos figurados do sangue, as plaquetas sofrem redução quantitativa em média de até $36 \%$ nos pacientes avaliados. Vários mecanismos são sugeridos para esse achado, entre eles, a invasão direta da medula óssea pelo SARS-CoV-2 e a destruição de clones precursores de trombócitos (QU $R$, et al., 2020). A invasão e inflamação do parênquima pulmonar também parecem contribuir; recentemente, o pulmão tem sido apontado como órgão liberador de megacariócitos (LEFRANÇAIS E, et al., 2017). A própria lesão pulmonar pelo excesso de $\mathrm{O} 2$ advindo dos altos fluxos dos respiradores mecânicos, constantemente usados nos pacientes, parece culminar em maior retenção plaquetária intrapulmonar, plaquetopenia na circulação periférica e aumento na taxa de ocorrência de eventos trombóticos, como o Tromboembolismo Pulmonar (TEP) (QU R, et al., 2020).

A infecção pelo SARS-CoV-2 também está associada a um estado pró-trombótico sistêmico, seja pela tempestade de citocinas decorrente das lesões celulares, seja pelo estado de desidratação (comum em pacientes debilitados), ou ainda pela imobilização no leito, pelas comorbidades associadas, pelo dano endotelial direto, pelos dispositivos invasivos como cateteres de acesso venoso ou tubo orotraqueal (TERPOS $\mathrm{E}$, et al., 2020). Todos esses elementos interagem no paciente gravemente enfermo e contribuem ainda mais para a plaquetopenia, desta vez por consumo; e também para o aumento dos marcadores inflamatórios e da coagulação, configurando mais um dos distúrbios hematológicos associados à COVID-19 (AHMED S, et al., 2020; HWA J, et al., 2020). Haverá, portanto, aumento das principais provas inflamatórias (proteína C reativa, pró-calcitonina, ferritina e desidrogenase lática); bem como alteração dos marcadores da coagulação como o D-dímero, consumo de fibrinogênio, alargamento do Tempo de Protrombina (TP) e do tempo de tromboplastina parcial ativada (TTPA) (LIPPI G e PLEBANI M, 2020). 
A dosagem do fibrinogênio e dos produtos de degradação da fibrina, em associação com níveis mais elevados de D-dímero, são encontrados em mais de $70 \%$ dos pacientes não sobreviventes em comparação com menos de $1 \%$ dos sobreviventes, estando relacionados com lesão miocárdica provocada pelo SARSCoV-2 (ZHANG L, et al., 2020).

Uma grande revisão de literatura com 58 artigos analisados sobre desordens autoimunes associadas à COVID-19, descreveu um total de 94 pacientes com resultados elucidativos quanto à epidemiologia dessas desordens. O estudo encontrou proporção muito semelhante entre homens e mulheres acometidos, sendo que a maioria dos relatos eram sobre pacientes acima de 60 anos, com somente 3 casos em pacientes pediátricos, tendo os 3 sido acometidos por Púrpura Trombocitopênica Imune (PTI). Cerca de $70 \%$ dos pacientes apresentavam alguma comorbidade anterior à COVID-19 e dentre os 94 pacientes avaliados, o distúrbio hematológico mais frequente foi a PTI com $58 \%$ das apresentações, seguida pela Anemia Hemolítica Autoimune (AHAl) presente em $23 \%$ dos casos (TAHERIFARD E, et al., 2021).

A mesma revisão ainda destacou que a Síndrome do Anticorpo Antifosolípide (SAF) pôde ser identificada em 10,6\% dos pacientes, a Púrpura Trombocitopênica Trombótica (PTT) e a Síndrome de Evans (SE) em $3,2 \%$ dos indivíduos, havendo apenas 1 caso de neutropenia autoimune. Quanto ao tempo de apresentação do quadro hematológico, verificou-se variação significativa entre as diversas condições com o menor tempo médio para a PTT e maior para PTI (4.6 e 13.3 dias, respectivamente). Dentre os 94 pacientes estudados, 88 permaneceram vivos ao final dos estudos e, dos que faleceram, a maioria fora acometida por AHAI (TAHERIFARD E, et al., 2021).

A SE é caracterizada como anemia hemolítica autoimune associada a trombocitopenia autoimune e, por vezes, neutropenia; podendo ser concomitantes ou surgindo de forma consecutiva na evolução da doença. Geralmente, há a associação de evidências laboratoriais de anemia hemolítica com Coombs direto positivo; além de apresentar, com avançar da história clínica, a plaquetopenia, associação essa que fecha os critérios para diagnóstico de SE (DALMASO BF, et al., 2019; BUSHAN S, et al., 2020). Também possui escassa descrição na literatura, considerando que o diagnóstico ocorre em cerca de 0,8-3,7\% dos pacientes que cursam com PTI ou AHAl; o que demonstra a raridade do fenômeno e a importância da descrição do crescente número de casos associados à infecção pelo SARS-CoV-2 (SUMER S, et al., 2020).

Durante a pandemia, o reconhecimento e condução da AHAl encontram ainda maior dificuldade, devido à grande variedade de achados hematológicos associados a COVID-19, a identificação da AHAI pode ser prejudicada ou mesmo superposta por outras condições como a PTT ou a SE. A polifarmácia e comorbidades associadas do paciente também são elementos de confusão diagnóstica para AHAI (KURNIAWAN A e ADELLA HD, 2019). Além disso, o tratamento com transfusão de hemocomponentes se encontra dificultado pela diminuição da disponibilidade de bolsas sanguíneas e a imunossupressão deve ser avaliada caso a caso para não propiciar estados ainda mais graves no paciente já enfermo (CONTENTTI EC e CORREA J, 2020; FAN BE, et al., 2020).

Apesar da insuficiência respiratória ser a principal causa de morte na COVID, a Coagulação Intravascular Disseminada (CIVD) e outros eventos trombóticos podem levar a uma rápida deterioração do quadro clínico, favorecendo desfechos sombrios. Um dos estudos mais citados sobre complicações trombóticas na COVID19 encontrou uma taxa de $0,6 \%$ de CIVD nos casos sobreviventes contra $71,4 \%$ em não sobreviventes, demonstrando a importância dessa condição na morbimortalidade da doença (TANG N, et al., 2020).

$\mathrm{Na}$ forma de supressão fibrinolítica da CIVD pela COVID-19, encontra-se uma associação fraca entre níveis aumentados de D-dímero e desfecho desfavorável, com esse marcador estando moderadamente alterado (ASAKURA H e OGAWA H, 2020). Alguns marcadores sanguíneos são significativamente diferentes dos encontrados nos quadros de CIVD por supressão fibrinolítica, como: Produto de Degradação da Fibrina (PDF) maior que $100 \mathrm{mcg} / \mathrm{ml}$, D-dímero de aproximadamente $20 \mathrm{mcg} / \mathrm{ml}$ e fibrinogênio por volta de 100 $\mathrm{mcg} / \mathrm{ml}$. Essa relação entre o D-dímero pouco aumentado com o PDF bastante elevado, aumenta o TP e não altera tanto o TTPA, não sendo condizente com a CIVD por supressão fibrinolítica da sepse, mas sim com CIVD por fibrinólise intensificada. Tal diferenciação demonstra que a CIVD do paciente com COVID-19 que leva à morte é, substancialmente, diferente da CIVD da sepse (TANG N, et al., 2020; ASAKURA H e OGAWA H, 2020; IBA T, et al., 2020). 
Uma possível explicação para essa característica diferenciada da CIVD foi elaborada por Hunt BJ e Levi M (2020) ao discutir a origem do D-dímero na COVID-19, sugerem que ao invés deste representar o produto da fibrinólise e ativação da coagulação in vivo, representaria o acúmulo dos produtos de fibrinólise principalmente das microtromboses pulmonares, sendo deslocados ao sangue. Tal fenômeno já fora descrito antes para ascites volumosas, hematomas e derrames pleurais, com deslocamento do D-dímero local para a circulação sistêmica. Mais estudos são necessários antes da recomendação de uso do D-dímero como marcador de lesão pulmonar, porém a explicação para a mudança do padrão esperado da CIVD pode passar por estas vias (ASAKURA H e OGAWA H, 2020).

Outra síndrome pró-trombótica reconhecida em pacientes com COVID-19 é a SAF, tratando-se de uma condição pró-trombótica associada à positividade dos anticorpos anticardiolipina, anti-beta-2 glicoproteína e do anticoagulante lúpico. É mais comum em mulheres jovens, podendo estar associada ao Lúpus Eritematoso Sistêmico (LES), outras desordens autoimunes e ao abortamento de repetição (SUNG J e ANJUM S, 2020). Em um estudo com 150 pacientes internados em unidade intensiva, 64 desenvolveram eventos tromboembólicos, sendo que a maioria desses pacientes apresentavam algum anticorpo antifosfolípide presente na circulação (HELMS J, et al., 2020).

Outra série de casos, com 3 pacientes com doença grave pelo SARS-CoV-2 e acometidos por eventos trombóticos, havia positividade dos anticorpos anticardiolipina e anti-beta-2 glicoproteina em todos os enfermos (MARIA ATJ, et al., 2020). Há também outros estudos demonstrando que mesmo em pessoas previamente saudáveis e sem fator de risco foram encontrados anticorpos antifosfolípide associados a eventos trombóticos como trombose venosa profunda e também em associação com o diagnóstico recente de LES durante a internação por COVID-19 (SUNG J e ANJUM S, 2020; MANTOVANI CE, et al., 2020).

Secundária à infecção pelo SARS-CoV-2, podem-se ainda identificar diversos fenômenos trombocitopênicos, dentre os quais destaca-se evento clínico de evolução semelhante a PTI; com paralelos descritos tanto na inadequada produção plaquetária medular, quanto na alteração no sistema imunológico cursando com destruição plaquetária em sangue periférico (HWA J, et al., 2020). Dentre os possíveis mecanismos de ação do SARS-CoV-2 sobre a plaquetopenia há a desregulação do microambiente medular, pela infecção direta de células medulares; a invasão e danos em parênquima pulmonar, afetando a liberação de megacariócitos e favorecendo sua atipia; a lesão hepática vírus mediada, reduzindo a produção de trombopoietina; além da desregulação imunológica, observada tanto pela tempestade de citocinas quanto invasão linfocitária, levando à destruição plaquetária imunomediada (AHMED S, et al., 2020).

Classicamente, a PTI possui um método de diagnóstico por exclusão de outras afecções que poderiam cursar com trombocitopenia; fato que pode levar a dificuldade de identificação da PTI como afecção secundária à infecção pelo SARS-CoV-2, necessitando de atenção especial durante a evolução clínica do paciente e nas variações dos marcadores laboratoriais, considerando a relação direta entre o pior prognóstico e presença de trombocitopenia (LAW L, et al., 2021; SOARES ACCV, et al., 2020).

Tendo em vista as alterações no estado do paciente grave ou de alto risco que predispõem a eventos tromboembólicos, fica evidente a necessidade de intervenção precoce a fim de minimizar tal risco (TANG N, et al., 2020). A mensuração contínua dos marcadores elencados é necessária para a correta avaliação dinâmica do estado do paciente e indicação da necessidade de cuidados anticoagulantes (AHMED S, et al., 2020; TERPOS E, et al., 2020). Um esquema de vigilância recomendado pela literatura inclui a necessidade de diagnóstico precoce e estratificação do risco individual para eventos tromboembólicos por meio da contagem de plaquetas, D-dímero, TP, TTPA e fibrinogênio; além do início precoce de anticoagulação profilática com Heparina não fracionada, heparina de baixo peso molecular ou drogas anticoagulantes orais (DOACs), como drogas de primeira linha para aqueles hospitalizados ou com alto risco de acordo com escores de avaliação vigentes (SPYROPOULOS AC, et al., 2020; TANG N, et al., 2020).

Mesmo pacientes não classificados como de alto risco para evento tromboembólico, parecem se beneficiar de avaliação constante, reservando atenção especial em casos de deterioração aguda dos sintomas, indicando-se nesses casos dosagem do D-dímero e avaliação por métodos de imagem como Angiotomografia computadorizada e Ultrassonografia com Doppler (AHMED S, et al., 2020; TERPOS E, et al., 2020). 


\section{CONSIDERAÇÕES FINAIS}

A infecção pelo SARS-CoV-2, no contexto pandêmico, ainda configura como um desafio à saúde pública mundial, haja vista sua rápida dispersão e grande variedade de repercussões orgânicas e sistêmicas. Dentre as principais apresentações da doença, os distúrbios hematológicos e da autoimunidade têm papel importante na morbimortalidade, principalmente quando associados aos eventos tromboembólicos. Desta forma, a presente revisão discute com a comunidade acadêmica, a necessidade de reconhecer precocemente as principais alterações hematológicas autoimunes que podem ser desencadeadas durante ou após o quadro agudo viral.

\section{REFERÊNCIAS}

1. AHMED S, et al. Thrombosis in Coronavirus disease 2019 (COVID-19) through the prism of Virchow's triad. Clin Rheumatol, 2020; 11: 1-15.

2. ASAKURA H, OGAWA H. COVID-19-associated coagulopathy and disseminated intravascular coagulation International. Journal of Hematology, 2020; 113(1): 45-57.

3. BUSHAN S, et al. Evans syndrome in a patient with COVID-19. British Journal of Haematology, 2020; 190: 59-61.

4. CHANNAPPANAVAR R, PERLMAN S. Pathogenic human coronavirus infections: causes and consequences of cytokine storm and immunopathology. Semin Immunopathol, 2017; 39(5): 529-539.

5. CONTENTTI EC, CORREA J. Immunosuppression during the COVID-19 pandemic in neuromyelitis optica spectrum disorders patients: a new challenge. Mult Scler Relat Disord, 2020; 41: 1-2.

6. DALMASO BF, et al. Síndrome de Evans em paciente lúpico do sexo masculino: relato de caso. J Bras Patol Med Lab, 2019; 55(4): 416-425.

7. FAN BE, et al. Hematologic parameters in patients with COVID-19 infection. Am J Hematol, 2020; 95(6): 131-134.

8. HELMS J, et al. High risk of thrombosis in patients with severe SARS-CoV-2 infection: a multicenter prospective cohort study. Intensive Care Med, 2020; 46(6):1089-1098.

9. HUNT BJ, LEVI M. Re the source of elevated plasma D-dimer levels in COVID-19 infection. Br J Haematol, 2020; 190(3): 133-134.

10. HWA J, et al. Thrombocytopathy and endotheliopathy: crucial contributors to COVID-19 thromboinflammation. Springer Nature Limited, 2020; 18: 194-209.

11. IBA T, et al. The unique characteristics of COVID-19 coagulopathy. Crit. Care, 2020; 24.

12. KURNIAWAN A, HALIM DA. Management of Autoimmune Hemolytic Anemia in the Midst of Coronavirus Disease 2019 Pandemic: A Case Report. Medicinus, 2019; 7(7): 223-228.

13. LAW L, et al. Atypical manifestations of coronavirus disease 2019 (COVID-19) - associated autoimmune thrombotic thrombocytopenic purpura. Clin Case Rep, 2021; 9(3): 1402-1404.

14. LEFRANÇAIS E, et al. The lung is a site of platelet biogenesis and a reservoir for haematopoietic progenitors. Nature, 2017; 544(7648):105-109.

15. LIN L, et al. Hypothesis for potential pathogenesis of SARS-CoV-2 infection-a review of immune changes in patients with viral pneumonia. Emerg Microbes Infect, 2020; 9(1): 727-732.

16. LIPPI G, PLEBANI M. Laboratory abnormalities in patients with COVID-2019 infection. Clin Chem Lab Med, 2020; 58(7): 1131-1134.

17. LIPPI G, PLEBANI M. The critical role of laboratory medicine during coronavirus disease 2019 (COVID-19) and other viral outbreaks. Clin Chem Lab Med, 2020; 58(7): 1063-1069.

18. MANTOVANI CE, et al. Concomitant new diagnosis of systemic lúpus erythematosus and COVID-19 with possible antiphospholipid syndrome. Just a coincidence? A case report and review of intertwining pathophysiology. Clin Rheumatol, 2020; 39(9): 2811-2815.

19. MARIA ATJ, et al. Flare of antiphospholipid syndrome in the course of COVID-19. TH Open, 2020; 4(3): $207-210$.

20. MEHTA P, et al. COVID-19: consider cytokine storm syndromes and immunosuppression. Lancet, 2020; 395(10229): 1033-1034.

21. ORGANIZAÇÃO MUNDIAL DA SAÚDE (OMS). COVID-19 Weekly Epidemiological Update: data as received by WHO from national authorities, as of 17 October 2021. 2021; Edição 62. Disponível em: https://www.who.int/publications/m/item/weekly-epidemiological-update-on-covid-19---19-october-2021. Acessado em: 19 de outubro de 2021.

22. QU R, et al. Platelet-to-lymphocyte ratio is associated with prognosis in patients with coronavirus disease-19. Journal Med Virol, 2020; 92(9): 1533-1541.

23. RAHI MS, et al. Hematologic disorders associated with COVID-19: a review. Annals of Hematology, 2020; 100(2): 309320. 
24. SCHULMAN S. Coronavirus Disease 2019, Prothrombotic Factors, and Venous Thromboembolism. Semin Thromb Hemost, 2020; 46(7): 772-776.

25. SMADJA DM, DEBUC B. Is COVID-19 a New Hematologic Disease? Stem Cell Reviews and Reports, 2021 ; 5: 1-5.

26. SOARES ACCV, et al. Outcome of SARS-CoV-2 and immune thrombocytopenia in a pediatric patient. Hematol transfus cell ther, 2021; 43(1): 101-103.

27. SPYROPOULOS AC, et al. Modified IMPROVE VTE risk score and elevated D-Dimer identify a high venous thromboembolism risk in acutely ill medical population for extended thromboprophylaxis. TH Open, 2020; 4(1): 59-65.

28. SUMER S, et al. A case of Evans syndrome secondary to COVID-19. Blood. Transfus, 2021; 19(1): 85-88.

29. SUNG J, ANJUM S. Coronavirus disease 2019 (COVID-19) infection associated with antiphospholipid antibodies and four-extremity deep vein thrombosis in a previously healthy female. Cureus, 2020; 12(6): 8408.

30. TAHERIFARD E, et al. Hematologic autoimmune disorders in the course of COVID-19: a systematic review of reported cases. Hematology, 2021; 26(1): 225-239.

31. TANG N, et al. Anticoagulant treatment is associated with decreased mortality in severe coronavirus disease 2019 patients with coagulopathy. J Thromb Haemost, 2020; 18(5): 1094-1099.

32. TERPOS E, et al. Hematological findings and complications of COVID-19. Am J Hematol, 2020; 95(7): 834-847.

33. VARGA Z, et al. Endothelial cell infection and endotheliitis in COVID-19. Lancet Lond Engl., 2020; 395(10234): 14171418.

34. WANG D, et al. Clinical characteristics of 138 hospitalized patients with 2019 novel coronavirus-infected pneumonia in Wuhan, China. JAMA, 2020; 323(11): 1061-1069.

35. XAVIER AR, et al. COVID-19: manifestações clínicas e laboratoriais na infecção pelo novo coronavírus. J Bras. Patol. Med. Lab, 2020; 56.

36. XU H, et al. High expression of ACE2 receptor of 2019-nCoV on the epithelial cells of oral mucosa. Int J Oral Sci, 2020; 12(1).

37. ZHANG L, et al. D-dimer levels on admission to predict in hospital mortality in patients with Covid-19. J Thromb Haemost JTH, 2020; 18(6): 1324-1329. 